\title{
Palmar and plantar lichen planus: a case report and review of the literature*
}

\author{
Ana Maria Abreu Velez ${ }^{1}$ \\ Neville Pereyo ${ }^{2}$
}

\author{
Michael S Howard ${ }^{1}$
}

DOI: http:/ / dx.doi.org/10.1590/abd1806-4841.20153034

\begin{abstract}
A bstract: Palmoplantar lichen planus is an uncommon dermatosis. We present a case of 38-year-old Caucasian male with a history of pruritic, scaly lesions on the right plantar foot. Physical examination revealed whitish plaques and numerous spiny hyperkeratotic papules and focal scaling. A biopsy demonstrated orthohyperkeratosis and acanthosis of the epidermis. Immunohistochemical staining revealed positivity within the epidermis and/ or lichenoid infiltrate with CD3, CD8, CD45, CD68, myeloid histiod antigen, BCL2, p27, p53, HLA-DPDQDR, metallothionein and tissue inhibitor of metalloproteinases 1. The diagnosis of PPLP was thus confirmed; this case illustrates that PPLP should be considered in the differential diagnosis of uncommon foot dermatoses with a significant junctional inflammatory component.
\end{abstract}

Keywords: Foot dermatoses; Lichen planus; Keratoderma, palmoplantar

\section{INTRODUCTION}

Palmoplantar lichen planus (PPLP) is an uncommon, localized variant of lichen planus (LP). ${ }^{1-4}$ Multiple clinical presentations of PPLP may be seen, the erythematous scaly form is most common and other clinical presentations include vesicle-like and petechia-like lesions, which are rare variants. ${ }^{1-4}$

\section{CASE RPORT}

A 38-year-old Caucasian male was evaluated for a rough, pruritic eruption of eight months' duration on the plantar right foot. He had been treating with betamethasone dipropionate $0.05 \%$ cream twice daily for two weeks, with no improvement. He denied any additional skin eruptions, or mucosal, hair or nail lesions. He was not taking any oral medications. Physical examination revealed two areas of confluent, erythematous, keratotic papules and plaques with scaling on the lateral proximal aspect and the medial plantar arch of the right foot. A biopsy for hematoxylin and eosin (H\&E) staining, as well as immunohistochemical (IHC) analysis was performed. H\&E examination, the epidermal stratum granulosum was accentuated and no epidermal acantholysis or epidermolytic hyperkeratosis was seen. Orthohyperkeratosis and acanthosis was noted within the epidermis, but no papillomatosis. "Sawtooth" rete ridges were present, and a lichenoid lymphohistiocytic infiltrate was noted along the basement membrane zone (BMZ) junction. Focal epidermal atrophy was seen with rarefaction of the BMZ. In addition, occasional necrotic keratynocytes (Civatte bodies) were present (Figure 1). No subepidermal blister (Max

Received on 05.08.2013.

Approved by the Advisory Board and accepted for publication on 12.08.2014.

Work performed at the Funding: Georgia Dermatopathology Associates - Atlanta, USA.

Financial Support: None.

Conflicts of Interest: None.

Georgia Dermatopathology Associates - Atlanta, USA.

Dermatology and Skin Surgery Center - Stockbridge, USA.

(C)2015 by Anais Brasileiros de Dermatologia 

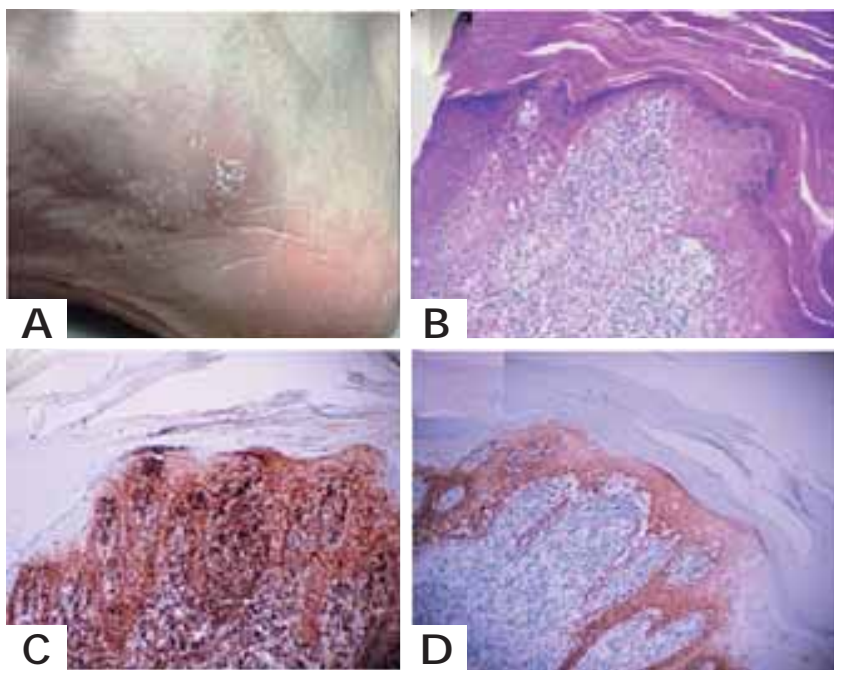

FIgure 1: Clinical and immunohistochemistry studies. 1A. A clinical picture highlighting confluent, erythematous, keratotic papules and plaques with scaling on the lateral proximal aspect and the medial plantar arch of the foot. 1B H\&E staining reveals orthohyperkeratosis and epidermal atrophy with a dense, lymphohistiocytic infiltrate located under the BMZ. 1C. IHC staining with anti-HLA-DPDQDR, strongly positive in the epidermis as well as in the subjacent dermal infiltrate (brown staining). 1D. IHC staining with anti-TIMP1, displaying positive staining in the epidermis, and negative staining where $\mathrm{BMZ}$ rarefaction is present (brown staining)

Joseph space) formation was noted. The constellation of clinical, histologic and special stain features was most representative of PPLP. IHC was performed as described before 5,6 and displayed positive staining in the inflammatory infiltrate with anti-human HLADP, DQ, DR antigen (Figure 1). Several spotty areas in the epidermis were positive for $\mathrm{p} 27$, as well as for myeloid histioid antigen (MHA). Tissue inhibitor of metalloproteinases 1(TIMP1), p53 and metallothionein all demonstrated significant positivity in lower areas of the epidermis (Figure 1). Staining for EGFR and matrix metalloproteinase 9 (MMP9) was negative. The lichenoid infiltrate subjacent to the BMZ was CD3, CD8, CD45, CD68 and BCL2 positive (Figure 2). CD4 stained was negative. The patient was subsequently treated with topical clobetasol propionate, $0.05 \%$ ointment, applied twice daily with improvement of the lesions.

\section{DISAUSSON}

PPLP is a rare localized variant of LP. ${ }^{1-4}$ Multiple LP variants have been documented, including actinic, annular, atrophic, bullous, hypertrophic, follicular, linear, nail, oral, and ulcerative LP. Another, uncommon variant of LP is PPLP. ${ }^{2-4}$ PPLP has been
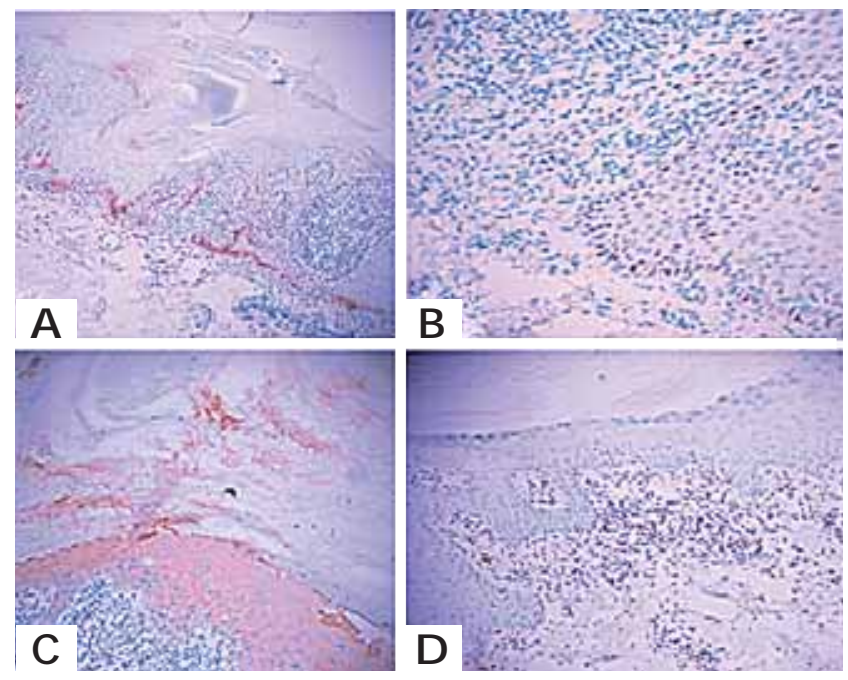

Figure 2: Immunohistochemistry stains. 2A. IHC staining with anti-metallothionein, demonstrating positivity along the BMZ (brown staining). 2B IHC staining with anti-p53, positive along the BMZ (brown staining). 2C. IHC staining with anti-p27, displaying positivity in the orthohyperkeratosis as well as in upper layers of the epidermis (brown staining). 2D. IHC staining with anti-BCL2, demonstrating positive staining in the lymphohistiocytic infiltrate located subjacent to the BMZ (brown staining)

reported with additional skin involvement in about $25 \%$ of cases. ${ }^{5,7}$ Classically, PPLP present with pruritic, erythematous, scaly and/or hyperkeratotic plaques with well-defined edges; in plantar cases, these lesions are often located on the internal arch, often without involvement of the palms or fingers. PPLP lesions usually disappear over a few months. ${ }^{1-3}$ Rare cases of PPLP have documented classic histologic features of LP. The clinical differential diagnosis includes tinea manum/pedis and psoriasis; moreover, PPLP lesions, in contrast to classic LP lesions, classically do not display Wickham's striae and are not shin. The clinical differential diagnosis also includes spiny keratoderma, keratoderma, dyshidrotic eczema and mycosis fungoides. An important study help to define PPLP by having the presence of pruriginous erythematous scaly and/or hyperkeratotic plaques, with well-defined edges, located on the internal plantar arch, lacking involvement of the fingertips, and which usually disappear in a few months. Histopathological features are similar to LP. ${ }^{8}$

In the current case, the histologic differential diagnosis includes a chronic lichenoid drug reaction, despite the paucity of eosinophils observed. Contrary to many cases of LP, our EGFR staining was negative; however, consistent 
with an LP pattern, p53 was positive in the BMZ. Contrary to oral LP, our MMP9 staining was negative differing from other oral LP study. ${ }^{9}$ As in oral LP, we found significant damage to the basal cell layer; this histologic feature is a characteristic histologic feature that may be associated with cell repair and cell death. p27 is a cell proliferation activity marker, and a cyclin-dependent kinase inhibitor involved in G1 cell cycle arrest. As demonstrated in some cases of oral LP, our p27 marker was positive, and thus may play a pivotal role in the control of cell proliferation and the expression of cell cycle regulatory proteins in PPLP. ${ }^{10}$

The observed positive staining for HLA-DPDQDR in lesional areas may be indicative of an active presentation of peptides to $\mathrm{T}$ lymphocytes; these $\mathrm{T}$ lymphocytes would be present with our findings of CD3, CD8 and CD45 positive infiltrate. Further evidence of an active antigen presentation process within and near the infiltrate is the observed positivity for two additional antigen presenting cell markers (CD68 and MHA). Several previous studies have shown that LP is an autoimmune skin disease, and our findings point towards a $\mathrm{T}$ cell suppressor/cytotxic response in contradistinction to T helper response. Also of interest are the positive findings of $\mathrm{Bcl}-2$ protein expression, associated with p27 and p53 protein expressions. Recent experimental studies have shown that $\mathrm{Bcl}-2$ (which has been established as a key player in the control of apoptosis) plays an important role in regulating cell cycles and cellular proliferation. In addition, the positive findings of p27 and p53 protein expression correlate with the fact that in PPLP, the cell cycle is altered (as suggested by the nature of these cell turnover markers). Thus, we suggest that in PPLP, active antigen presentation and a resultant immune response occurs, as well as alterations in immune system cellular proliferation rates. We also speculate that simultaneous with these events, metallothionein and TIMP1 production begins to affect tissue inflammation and remodeling.

We conclude that PPLP is a rare disease that can be easily misdiagnosed. PPLP may present only on the palms and/or soles; alternatively, it may also present with other skin lesions. Histologic examination assists in the diagnosis; IHC staining contributes not only to the diagnosis, but also to our understanding of the immunopathology of this disorder.

\section{REFERENCESS}

1. Landis M, Bohyer C, Bahrami S, Brogan B. Palmoplantar lichen planus: A rare presentation of a common disease. J Dermatol Case Rep. 2008;2:8-10.

2. Kim MJ, Choi M, Na SY, Lee JH, Cho S. Two cases of palmoplantar lichen planus with various clinical features. J Dermatol. 2010;37:985-9.

3. Mehta V, Vasanth V, Balachandran C. Palmar involvement in lichen planus. Dermatol Online J. 2009;15:12.

4. Rotunda AM, Craft N, Haley JC. Hyperkeratotic plaques on the palms and soles. Palmoplantar lichen planus, hyperkeratotic variant. Arch Dermatol. 2004; 140:1275-80.

5. Abreu Velez AM, Yi H, Googe PB Jr, Mihm MC Jr, Howard MS. Autoantibodies to melanocytes and characterization of melanophages in patients affected by a new variant of endemic pemphigus foliaceus. J Cutan Pathol. 2011;38:710-9.

6. Abreu Velez AM, Yi H, Gao W, Smoller BR, Grossniklaus HE, Howard MS Antibodies to pilosebaceous units along their neurovascular supply routes in a new variant of endemicpemphigus foliaceus in Colombia, South America. Eur J Dermatol. 2011;21:371-5

7. Boyd AS, Neldner KH. Lichen planus. J Am Acad Dermatol. 1991:25:593-619.

8. Sánchez-Pérez J, Rios Buceta L, Fraga J, García-Díez A. Lichen planus with lesions on the palms and/or soles: prevalence and clinicopathological study of 36 patients. Br J Dermatol. 2000;142:310-4.

9. Paulusová V, Laco J, Drízhal I, Slezák R. Expression of matrix metalloproteinase 9 in patients with oral lichen planus. Acta Medica (Hradec Kralove). 2012;55:23-6.

10. Hirota M, Ito T, Okudela K, Kawabe R, Yazawa T, Hayashi H, et al. Cell proliferation activity and the expression of cell cycle regulatory proteins in oral lichen planus. J Oral Pathol Med. 2002;31:204-12.

\author{
M AILING ADDRESS: \\ A na M aria A breu Velez \\ Georgia D ermatopathology A ssociates, 1534 \\ N orth D ecatur Rd., NE; Suite 206; \\ A tlanta, Georgia 30307-1000, U SA \\ E-mail: abreuvelez@yahoo.com
}

H ow to cite this article: Abreu-Velez AM, Howard MS, Pereyo N. Palmoplantar lichen planus: a case report and review of the literature. An Bras Dermatol. 2015;90(3 Suppl 1):S175-7. 